Original Article

\title{
Molecular and epidemiological analysis of IMP-1 metallo- $\beta$ - lactamase-producing Klebsiella pneumoniae in a tertiary care hospital in Japan
}

\author{
Hiromi Yamakawa a, b, Kosuke Kosai a, *, Norihiko Akamatsu ${ }^{\text {a }}$, Junichi Matsuda ${ }^{\text {a }}$ \\ Norihito Kaku ${ }^{a}$, Naoki Uno ${ }^{b}$, Yoshitomo Morinaga ${ }^{b}$, Hiroo Hasegawa ${ }^{a}$,

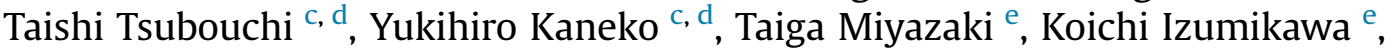 \\ Hiroshi Mukae $^{\mathrm{f}}$, Katsunori Yanagihara ${ }^{\mathrm{a}, \mathrm{b}}$ \\ a Department of Laboratory Medicine, Nagasaki University Hospital, Nagasaki, Japan \\ b Department of Laboratory Medicine, Nagasaki University Graduate School of Biomedical Sciences, Nagasaki, Japan \\ ${ }^{c}$ Department of Bacteriology, Osaka City University Graduate School of Medicine, Osaka, Japan \\ ${ }^{\mathrm{d}}$ Research Center for Infectious Disease Sciences, Osaka City University Graduate School of Medicine, Osaka, Japan \\ e Department of Infectious Diseases, Nagasaki University Graduate School of Biomedical Sciences, Nagasaki, Japan \\ ${ }^{\mathrm{f}}$ Department of Respiratory Medicine, Nagasaki University Graduate School of Biomedical Sciences, Nagasaki, Japan
}

\section{A R T I C L E I N F O}

\section{Article history:}

Received 21 April 2018

Received in revised form

13 November 2018

Accepted 30 November 2018

Available online 2 January 2019

\section{Keywords:}

Carbapenemase

MLST

Risk factors

\begin{abstract}
A B S T R A C T
This study investigated the molecular and phenotypic characteristics of carbapenemase-producing Klebsiella pneumoniae, and identified the risk factors underlying its acquisition. We evaluated K. pneumoniae isolated in Nagasaki University Hospital between January 2009 and June 2015. The presence of carbapenemase genes and plasmid characteristics were investigated. We performed multilocus sequence typing (MLST), and generated a dendrogram based on the results of pulsed-field gel electrophoresis (PFGE) for carbapenemase-producing strains. We also performed a case-control study of patients. Of the $88 \mathrm{~K}$. pneumoniae strains that showed minimum inhibitory concentration $\geq 1 \mu \mathrm{g} / \mathrm{mL}$ for imipenem and/or meropenem, and that were available from our bacterial collection, 18 had the IMP-type carbapenemase gene, all of which were IMP-1 according to sequencing analysis. Strains included seven different sequence types (STs), of which the most common was ST1471. A dendrogram showed the significant similarity of some strains with relationships in PFGE patterns, STs, and the wards in which they were isolated. Plasmid incompatibility group was similar among the IMP-1 producers. Regarding risk factors, multivariate analysis showed that liver disease and previous uses of carbapenems and anti-fungal drugs were significant factors for the acquisition of IMP-1-producing strains. Our results demonstrate that IMP-1 is a major carbapenemase produced by K. pneumoniae. The PFGE results indicated the possibility of transmission in the hospital. The identified risk factors should be considered for appropriate antibiotic therapy and infection-control measures.
\end{abstract}

(c) 2018 Japanese Society of Chemotherapy and The Japanese Association for Infectious Diseases. Published by Elsevier Ltd. All rights reserved.

\section{Introduction}

Klebsiella pneumoniae is an important pathogen of various infections such as pneumonia, biliary and urinary tract infections, and bacteremia [1-4]. Until recently, carbapenems were one of the

\footnotetext{
* Corresponding author. Department of Laboratory Medicine, Nagasaki University Hospital 1-7-1 Sakamoto, Nagasaki, 852-8501, Japan

E-mail address: k-kosai@nagasaki-u.ac.jp (K. Kosai).
}

most reliable antimicrobials for treating infections caused by $K$. pneumoniae including extended-spectrum $\beta$-lactamase-producing strains; however, the prevalence of carbapenem-resistant K. pneumoniae is increasing worldwide. Plasmid-mediated carbapenemase producers are especially problematic because plasmids harboring resistant genes transfer among different bacterial genera or species [5-7].

There are many types of carbapenemases found on plasmids such as K. pneumoniae carbapenemases (KPCs), OXA- $\beta$-lactamases, 
and metallo- $\beta$-lactamases (MBLs) including New Delhi MBL (NDM), Verona integron-encoded MBL (VIM), and IMP-type MBL $[8,9]$. The outbreaks by KPC-, NDM-, and VIM-type carbapenemase-producers have been reported in Europe and KPCs are the most prevalent carbapenemases in the United States [5,6]. In Japan, IMP-type MBLs, especially IMP-1, are widespread [10-12].

The Japan Nosocomial Infections Surveillance (JANIS), a program of the Ministry of Health Labour and Welfare, reported in 2016 that $0.2 \%$ and $0.5 \%$ of $K$. pneumoniae are resistant to imipenem and meropenem according to the Clinical and Laboratory Standard Institute (CLSI) definitions, respectively [13]. These resistant rates for carbapenems are not currently high. However, in addition to dissemination of IMP-1, IMP-6-producing K. pneumoniae, which are susceptible to imipenem but resistant to meropenem, have newly emerged in recent years in the western part of Japan [14]. Furthermore, a recent report noted that the resistance rates to imipenem and meropenem in Enterobacteriaceae are stably increasing and that Klebsiella spp. accounted for the largest population of carbapenemresistant Enterobacteriaceae (CRE) in Asia [7].

In contrast to European countries and the United States, the epidemiological characteristics of carbapenemase-producing $K$. pneumoniae have not been fully investigated in Japan. Therefore, we conducted molecular and phenotypic analyses of carbapenemase-producing $K$. pneumoniae isolated in our hospital. Additionally, we performed a case-control study of patients to identify risk factors for the acquisition of carbapenemaseproducing K. pneumoniae.

\section{Patients and methods}

\subsection{Screening of bacterial strains}

We retrospectively investigated $K$. pneumoniae isolated in Nagasaki University Hospital between January 2009 and June 2015. Strains for which minimum inhibitory concentrations (MICs) were $\geq 1 \mu \mathrm{g} / \mathrm{mL}$ for imipenem and/or meropenem were extracted from the database for further analyses. We measured MICs using the BD Phoenix Automated Microbiology System (BD Diagnostics). Drug susceptibility results were interpreted according to the CLSI M100S28 [15]. For case-control studies, if strains were repeatedly identified from a single patient during the study period, de-duplication was performed and the first isolated strain was included. This process was performed regardless of specimen type.

\subsection{Detection of carbapenemase genes and production}

We used PCR to evaluate the presence of IMP and KPC carbapenemase genes for strains with MIC $\geq 1 \mu \mathrm{g} / \mathrm{mL}$ for imipenem and/ or meropenem. DNA was extracted using the boiling method. Bacterial colonies were suspended in $100 \mu \mathrm{L}$ Tris-EDTA buffer containing $250 \mathrm{U} / \mathrm{mL}$ achromopeptidase (Wako Pure Chemical Industries, Ltd.), and were incubated at $50^{\circ} \mathrm{C}$ for $10 \mathrm{~min}$. After adding $250 \mu \mathrm{L}$ of $10 \%$ Chelex $^{\circledR} 100$ Resin (Bio-Rad), the suspension was boiled at $99^{\circ} \mathrm{C}$ for $5 \mathrm{~min}$, cooled on ice for $1 \mathrm{~min}$, and centrifuged at 12,000 rpm for $1 \mathrm{~min}$. Then the supernatant was harvested and used as a template for PCR amplification. PCR primers to amplify IMP and KPC genes were as follows: IMP forward, 5'-GGAATAGAGTGGCTTAAYTCTC-3'; IMP reverse, 5'-GGTTTAAYAAAACAACCACC-3'; KPC forward, 5'CGTCTAGTTCTGCTGTCTTG-3'; KPC reverse, 5'-CTTGTCATCCTTGTTAGGCG-3' [2]. PCR amplification was performed under the following conditions: $10 \mathrm{~min}$ at $94^{\circ} \mathrm{C}, 40$ cycles consisting of $30 \mathrm{~s}$ at $94{ }^{\circ} \mathrm{C}, 40 \mathrm{~s}$ at $52{ }^{\circ} \mathrm{C}, 1 \mathrm{~min}$ at $72{ }^{\circ} \mathrm{C}$, and $5 \mathrm{~min}$ at $72{ }^{\circ} \mathrm{C}$ for the final extension. DNA fragments were analyzed by electrophoresis on a $1.5 \%$ agarose gel. If IMP-type MBL was detected, direct sequencing was performed using the ABI PRISM 3130 Genetic Analyzer according to the manufacturer's instructions (Applied Biosystems) to differentiate between IMP-1 and IMP-6, which only differed by one base pair compared to IMP-1 [16]. Carbapenemase production was examined using the modified carbapenem inactivation method (mCIM) according to the CLSI M100-S28 [15].

\subsection{Analysis of plasmid characteristics}

Plasmid incompatibility groups were determined using a PCRbased replicon typing kit (Diatheva), which can detect 25 major replicons by 8 multiplex PCRs, according to the manufacturer's instructions. Also, conjugal transfer of plasmid was evaluated. For bacterial conjugation, carbapenem-sensitive but quinoloneresistant Escherichia coli was used as a recipient. IMP-1-producing $K$. pneumoniae and a recipient were coincubated in LB broth at $30{ }^{\circ} \mathrm{C}$ for $12-18 \mathrm{~h}$ and inoculated onto LB agar containing ciprofloxacin $(35 \mu \mathrm{g} / \mathrm{mL})$ and meropenem $(25 \mu \mathrm{g} / \mathrm{mL})$. After overnight incubation, bacteria grown were confirmed to be $E$. coli and the presence of IMP genes was evaluated by PCR.

\subsection{Multilocus sequence typing}

We performed multilocus sequence typing (MLST) for carbapenemase-producing strains, targeting seven housekeeping genes (rpoB, gapA, mdh, pgi, phoE, infB, and tonB) as previously described [17]. The allele sequences and sequence types (STs) were determined according to the Klebsiella MLST database (http://bigsdb. pasteur.fr/klebsiella/klebsiella.html).

\subsection{Pulsed-field gel electrophoresis}

Pulsed-field gel electrophoresis (PFGE) using the XbaI restriction enzyme was performed by Miroku Medical Laboratory Inc. according to the manufacturer's instructions and as described in the textbook with modifications [18]. A Dice coefficient-based dendrogram was generated according to the unweighted-pair group method with arithmetic mean (UPGMA) using Fingerprinting II Software Version 3.0 (Bio-Rad). The chromosomal DNA restriction patterns were interpreted according to the criteria as previously reported [19].

\subsection{Case-control study}

We conducted a matched case-control study using a 1:3 case to control ratio to identify risk factors for the acquisition of carbapenemase-producing K. pneumoniae. Control patients (uninfected control) were selected among patients from whom carbapenemase producers had not been detected. The following matching parameters were used to select uninfected control: ward, year and month, and period at risk. The period at risk was defined as duration from admission to isolation of carbapenemase-producing strain for case patients, and as the total length of hospital stay for control patients, which was at least as long as that for the matched case patient. If the number of control patients who met the criteria was less than three patients because the length of hospital stay was shorter than the period at risk for the matched case, we selected controls in descending order of the length of hospital stay. If four or more patients for each case met the matching criteria, we selected control patients by random sampling using Excel (Microsoft Corporation). We chose this method to select control patients because previous reports have stated that this method provides the appropriate risk factors in a case-control study related to the acquisition of resistant bacteria [20-22]. We compared patient backgrounds between the case and control groups. We also analyzed the risk factors for acquiring carbapenemase-producing strains. 


\subsection{Statistical analysis}

Matched univariate and multivariate analyses were performed using the conditional logistic regression model. Variables regarding comorbidities/conditions, use of medical devices, and prior antibiotic use with $\mathrm{P}$ values less than 0.05 in the univariate analysis were selected and adjusted by backward stepwise selection in the multivariate analysis to identify risk factors for acquisition of carbapenemase-producing strains. Data were analyzed using IBM SPSS statistics version 20 , and $\mathrm{P}$ values of 0.05 were considered statistically significant.

\section{Results}

\subsection{Bacterial strains, drug susceptibility, and carbapenemase detection}

Of the $1462 \mathrm{~K}$. pneumoniae strains isolated during the study period, 120 showed MIC $\geq 1 \mu \mathrm{g} / \mathrm{mL}$ for imipenem and/or meropenem, and 88 strains were available from our bacterial collection. We detected IMP-type MBL gene in 18 strains among the 88 strains examined. No KPC gene was detected. Sequencing analysis revealed that the 18 strains had the IMP-1-MBL gene but not the IMP-6 gene. Carbapenemase production was confirmed by $\mathrm{mCIM}$ in the 18 strains. The number of isolates peaked in 2012 (8 strains) and decreased thereafter. The results of drug susceptibilities are presented in Table 1. Although no strain was susceptible to imipenem, one was susceptible to meropenem. The MICs for amikacin were $\leq 4 \mu \mathrm{g} / \mathrm{mL}$ in all 18 strains. Only two strains were resistant to ciprofloxacin according to the CLSI definitions [15].

\subsection{Multilocus sequence typing}

We submitted the new ST to the Klebsiella MLST database, which was defined as ST2603 (http://bigsdb.pasteur.fr/klebsiella/ klebsiella.html). Strains were divided into seven different STs by MLST (Fig. 1), of which the most frequent type was ST1471 (six strains), followed by ST1148, ST1484, and ST2603 (three strains, respectively).

\subsection{Pulsed-field gel electrophoresis}

The similarity among strains was analyzed by a dendrogram based on the PFGE results. Three strains (KP15, KP16, and KP17) and three strains (KP1, KP3, and KP6) showed >80\% similarity, respectively; and three strains (KP11, KP12, and KP14), two strains (KP1 and KP3), and six strains (KP4, KP5, KP7, KP8, KP9, and KP10) showed complete similarity (100\%), respectively (Fig. 1). We found relationships among PFGE patterns, STs, and the wards in which they were isolated, indicating the possibility that several strains were transmitted in the hospital.

\subsection{Plasmid characteristics}

IncL was the unique incompatibility group detected in the 17 IMP-producing K. pneumoniae. We could not determine the incompatibility group in one strain (KP13). Before the evaluation of conjugal transfer, all 18 IMP-producing $K$. pneumoniae and the recipient were cultured on LB agar containing only $35 \mu \mathrm{g} / \mathrm{mL}$ of ciprofloxacin. The recipient grew but 17 IMP-producing K. pneumoniae including KP10, which was a ciprofloxacin-resistant strain, did not grow on it, that meant that only recipient could be effectively selected. However, because KP18 grew on the agar containing only $35 \mu \mathrm{g} / \mathrm{mL}$ of ciprofloxacin, the conjugal transfer was not evaluated for the strain. Plasmid transfer was confirmed in 17 IMP-producing K. pneumoniae other than KP18 by conjugation.

\subsection{Patient characteristics}

Table 2 shows details of 16 patients from whom IMP-1producing K. pneumoniae was isolated. The median age and median duration from admission to isolation were 51.5 years old (interquartile range, 6.0-62.0) and 35.5 days (interquartile range, 25.0-42.5), respectively. Eleven patients (68.8\%) were male. Eight strains were isolated from stool, followed by three strains from urine and ascites, and two strains from tracheal tube. One isolate (KP17) was detected by active screening. When the IMP-1 producer was isolated, nine patients were hospitalized in the A ward, five patients were in the B ward, and two patients were in the intensive care unit (ICU). Two patients from whom strains were isolated only

Table 1

Results of the drug susceptibility of IMP-1 metallo- $\beta$-lactamase-producing Klebsiella pneumoniae.

\begin{tabular}{|c|c|c|c|c|c|c|c|c|c|c|c|c|c|c|c|c|c|c|c|c|}
\hline \multirow{3}{*}{$\begin{array}{l}\text { Strain } \\
\text { KP1 }\end{array}$} & \multirow{3}{*}{$\begin{array}{l}\text { Patient } \\
1\end{array}$} & \multirow{3}{*}{$\begin{array}{l}\text { Year } \\
2009\end{array}$} & \multicolumn{18}{|c|}{ MIC $(\mu \mathrm{g} / \mathrm{ml})$ and susceptibility interpretation } \\
\hline & & & \multicolumn{2}{|l|}{$\overline{\mathrm{TZP}}$} & \multicolumn{2}{|l|}{ CTX } & \multicolumn{2}{|l|}{ CAZ } & \multicolumn{2}{|l|}{ FEP } & \multicolumn{2}{|l|}{ IPM } & \multicolumn{2}{|l|}{ MEM } & \multicolumn{2}{|l|}{ CIP } & \multicolumn{2}{|c|}{ AMK } & \multicolumn{2}{|c|}{ GEN } \\
\hline & & & $16 / 4$ & $S$ & $>32$ & $\mathrm{R}$ & $>32$ & $\mathrm{R}$ & $>16$ & $\mathrm{R}$ & $>16$ & $\mathrm{R}$ & $>16$ & $\mathrm{R}$ & $\leq 0.5$ & $S$ & $\leq 4$ & $\mathrm{~S}$ & 4 & $S$ \\
\hline $\mathrm{KP} 2$ & 2 & 2010 & $8 / 4$ & S & 32 & $\mathrm{R}$ & $>32$ & $\mathrm{R}$ & 8 & SDD & 4 & $\mathrm{R}$ & 4 & $\mathrm{R}$ & $\leq 0.5$ & $S$ & $\leq 4$ & $\mathrm{~S}$ & $>8$ & $\mathrm{R}$ \\
\hline KP3 & 3 & 2010 & $32 / 4$ & I & $>32$ & $\mathrm{R}$ & $>32$ & $\mathrm{R}$ & $>16$ & $\mathrm{R}$ & 8 & $\mathrm{R}$ & 8 & $\mathrm{R}$ & 1 & $\mathrm{~S}$ & $\leq 4$ & $\mathrm{~S}$ & 8 & I \\
\hline KP4 & 4 & 2011 & $\leq 4 / 4$ & S & 32 & $\mathrm{R}$ & $>32$ & $\mathrm{R}$ & 16 & $\mathrm{R}$ & 4 & $\mathrm{R}$ & 2 & I & $\leq 0.5$ & $\mathrm{~S}$ & $\leq 4$ & $\mathrm{~S}$ & 8 & I \\
\hline KP5 & 5 & 2012 & $\leq 4 / 4$ & S & $>32$ & $\mathrm{R}$ & $>32$ & $\mathrm{R}$ & 16 & $\mathrm{R}$ & 2 & I & 4 & $\mathrm{R}$ & $\leq 0.5$ & $S$ & $\leq 4$ & $\mathrm{~S}$ & 8 & I \\
\hline KP6 & 6 & 2012 & $32 / 4$ & I & $>32$ & $\mathrm{R}$ & $>32$ & $\mathrm{R}$ & $>16$ & $\mathrm{R}$ & $>16$ & $\mathrm{R}$ & $>16$ & $\mathrm{R}$ & $\leq 0.5$ & $\mathrm{~S}$ & $\leq 4$ & $\mathrm{~S}$ & 4 & $\mathrm{~S}$ \\
\hline KP7 & 7 & 2012 & $8 / 4$ & $S$ & $>32$ & $\mathrm{R}$ & $>32$ & $\mathrm{R}$ & 16 & $\mathrm{R}$ & 4 & $\mathrm{R}$ & 4 & $\mathrm{R}$ & $\leq 0.5$ & $S$ & $\leq 4$ & $\mathrm{~S}$ & 8 & I \\
\hline KP8 & 8 & 2012 & $8 / 4$ & S & $>32$ & $\mathrm{R}$ & $>32$ & $\mathrm{R}$ & 16 & $\mathrm{R}$ & 4 & $\mathrm{R}$ & 4 & $\mathrm{R}$ & $\leq 0.5$ & $S$ & $\leq 4$ & $\mathrm{~S}$ & 8 & I \\
\hline KP9 & 9 & 2012 & $8 / 4$ & $S$ & $>32$ & $\mathrm{R}$ & $>32$ & $\mathrm{R}$ & $>16$ & $\mathrm{R}$ & $>16$ & $\mathrm{R}$ & $>16$ & $\mathrm{R}$ & $\overline{1}$ & $S$ & $\leq 4$ & $S$ & 8 & I \\
\hline KP10 & 9 & 2012 & $\leq 4 / 4$ & $\mathrm{~S}$ & $>32$ & $\mathrm{R}$ & $>32$ & $\mathrm{R}$ & 8 & SDD & 16 & $\mathrm{R}$ & $>16$ & $\mathrm{R}$ & $>4$ & $\mathrm{R}$ & $\leq 4$ & $\mathrm{~S}$ & 4 & $S$ \\
\hline KP11 & 10 & 2012 & $8 / 4$ & S & 32 & $\mathrm{R}$ & $>32$ & $\mathrm{R}$ & $>16$ & $\mathrm{R}$ & 8 & $\mathrm{R}$ & 4 & $\mathrm{R}$ & $\leq 0.5$ & $\mathrm{~S}$ & $\leq 4$ & $\mathrm{~S}$ & 8 & I \\
\hline KP12 & 11 & 2012 & $8 / 4$ & $S$ & 32 & $\mathrm{R}$ & $>32$ & $\mathrm{R}$ & 16 & $\mathrm{R}$ & 2 & I & 1 & $\mathrm{~S}$ & $\leq 0.5$ & $S$ & $\leq 4$ & $\mathrm{~S}$ & 8 & I \\
\hline KP13 & 6 & 2013 & $16 / 4$ & $S$ & $>32$ & $\mathrm{R}$ & $>32$ & $\mathrm{R}$ & $>16$ & $\mathrm{R}$ & 8 & $\mathrm{R}$ & 8 & $\mathrm{R}$ & $\leq 0.5$ & $S$ & $\leq 4$ & $\mathrm{~S}$ & 4 & $S$ \\
\hline KP14 & 12 & 2014 & $16 / 4$ & $\mathrm{~S}$ & $>32$ & $\mathrm{R}$ & $>32$ & $\mathrm{R}$ & 16 & $\mathrm{R}$ & 16 & $\mathrm{R}$ & 16 & $\mathrm{R}$ & $\leq 0.5$ & $\mathrm{~S}$ & $\leq 4$ & $\mathrm{~S}$ & 8 & I \\
\hline KP15 & 13 & 2014 & $\leq 4 / 4$ & $S$ & 32 & $\mathrm{R}$ & $>32$ & $\mathrm{R}$ & 8 & SDD & 4 & $\mathrm{R}$ & 4 & $\mathrm{R}$ & $\leq 0.5$ & $S$ & $\leq 4$ & $\mathrm{~S}$ & $>8$ & $\mathrm{R}$ \\
\hline KP16 & 14 & 2014 & $8 / 4$ & S & 32 & $\mathrm{R}$ & $>32$ & $\mathrm{R}$ & 8 & SDD & 4 & $\mathrm{R}$ & 4 & $\mathrm{R}$ & $\leq 0.5$ & S & $\leq 4$ & $S$ & 8 & I \\
\hline KP17 & 15 & 2015 & $16 / 4$ & S & $>32$ & $\mathrm{R}$ & $>32$ & $\mathrm{R}$ & $>16$ & $\mathrm{R}$ & 4 & $\mathrm{R}$ & 4 & $\mathrm{R}$ & $\overline{1}$ & $S$ & $\leq 4$ & $S$ & 8 & I \\
\hline KP18 & 16 & 2015 & $16 / 4$ & $\mathrm{~S}$ & $>32$ & $\mathrm{R}$ & $>32$ & $\mathrm{R}$ & $>16$ & $\mathrm{R}$ & 4 & $\mathrm{R}$ & 4 & $\mathrm{R}$ & $>4$ & $\mathrm{R}$ & $\leq 4$ & $\mathrm{~S}$ & 8 & I \\
\hline
\end{tabular}

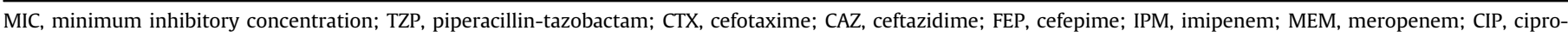
floxacin; AMK, amikacin; GEN, gentamicin; S, susceptible; I, intermediate; R, resistant; SDD, susceptible dose dependent. 
Similarity $(\%)$
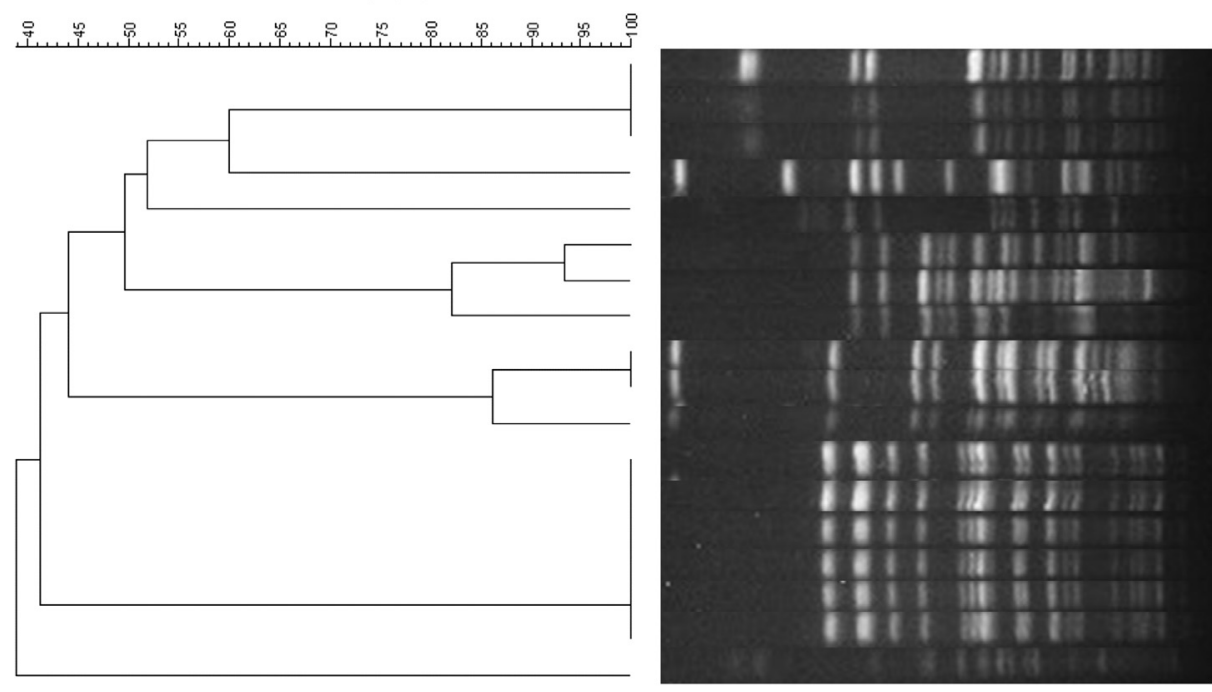

$\begin{array}{llll}\text { Strain } & \text { ST } & \text { Patient } & \text { Ward } \\ \text { KP11 } & 2603 & 10 & \text { B } \\ \text { KP12 } & 2603 & 11 & \text { B } \\ \text { KP14 } & 2603 & 12 & \text { B } \\ \text { KP2 } & 1757 & 2 & \text { A } \\ \text { KP18 } & 37 & 16 & \text { A } \\ \text { KP16 } & 1484 & 14 & \text { A } \\ \text { KP17 } & 1484 & 15 & \text { A } \\ \text { KP15 } & 1484 & 13 & \text { A } \\ \text { KP1 } & 1148 & 1 & \text { A } \\ \text { KP3 } & 1148 & 3 & \text { B } \\ \text { KP6 } & 1148 & 6 & \text { B } \\ \text { KP4 } & 1471 & 4 & \text { ICU } \\ \text { KP5 } & 1471 & 5 & \text { ICU } \\ \text { KP7 } & 1471 & 7 & \text { A } \\ \text { KP8 } & 1471 & 8 & \text { A } \\ \text { KP9 } & 1471 & 9 & \text { A } \\ \text { KP10 } & 1471 & 9 & \text { A } \\ \text { KP13 } & 443 & 6 & \text { B }\end{array}$

Fig. 1. A dendrogram of 18 IMP-1-producing Klebsiella pneumoniae generated based on the results of pulsed-field gel electrophoresis. ST, sequence type.

stayed in the ICU for 4 days, and had been hospitalized in the A ward before moving there.

Of the 16 patients from whom IMP-1-producing K. pneumoniae was isolated, five patients developed infection such as respiratory infection and intra-abdominal infection. Multiple pathogens were simultaneously isolated in all five patients (Table 2). Regarding treatment against infections caused by IMP-1 producers, patient 12 who developed respiratory infection was administered meropenem after treatment failure by ampicillin-sulbactam. Patients 2 and 13 with intra-abdominal infection received piperacillin-tazobactam.

Table 2

Clinical features of patients in whom IMP-1 metallo- $\beta$-lactamase-producing Klebsiella pneumoniae was isolated.

\begin{tabular}{|c|c|c|c|c|c|c|c|c|c|}
\hline Patient & Age & Sex & Ward & $\begin{array}{l}\text { Duration from } \\
\text { admission to } \\
\text { isolation (days) }\end{array}$ & $\begin{array}{l}\text { Isolation } \\
\text { site }\end{array}$ & Reason for admission & $\begin{array}{l}\text { Underlying diseases and } \\
\text { conditions on admission }\end{array}$ & $\begin{array}{l}\text { Clinical diagnosis } \\
\text { associated with IMP- } \\
\text { producing } K . \text { pneumoniae }\end{array}$ & $\begin{array}{l}\text { Simultaneously } \\
\text { isolated pathogens }\end{array}$ \\
\hline 1 & 37 & M & A & 28 & Stool & Liver transplantation & $\begin{array}{l}\text { Liver cirrhosis (chronic hepatitis } \\
\text { B and D), chronic kidney disease }\end{array}$ & Colonization & - \\
\hline 2 & 61 & $\mathrm{~F}$ & A & 2 & $\begin{array}{l}\text { Stool and } \\
\text { ascites }^{\mathrm{a}}\end{array}$ & Peritonitis & $\begin{array}{l}\text { After liver transplantation due to } \\
\text { liver cirrhosis (chronic hepatitis C), } \\
\text { Diabetes mellitus }\end{array}$ & Peritonitis & $\begin{array}{l}\text { Enterococcus faecium } \\
\text { from ascites }\end{array}$ \\
\hline 3 & 4 & M & B & 28 & Stool & Medulloblastoma & $\begin{array}{l}\text { Symptomatic epilepsy, } \\
\text { developmental delay }\end{array}$ & Colonization & - \\
\hline 4 & 50 & M & ICU & 12 & Stool & Liver transplantation & $\begin{array}{l}\text { Liver cirrhosis } \\
\text { (chronic hepatitis C) }\end{array}$ & Colonization & - \\
\hline 5 & 53 & M & ICU & 35 & Stool & Liver transplantation & Liver cirrhosis & Colonization & \\
\hline 6 & 1 & M & B & 40 & $\begin{array}{l}\text { Tracheal } \\
\text { tube }\end{array}$ & Sepsis & None & Colonization & - \\
\hline 7 & 55 & $\mathrm{~F}$ & A & 22 & Stool & Liver transplantation & $\begin{array}{l}\text { Liver cirrhosis } \\
\text { (chronic hepatitis C) }\end{array}$ & Colonization & - \\
\hline 8 & 65 & M & A & 32 & Stool & Liver transplantation & $\begin{array}{l}\text { Hepatocellular carcinoma, } \\
\text { alcoholic liver cirrhosis }\end{array}$ & Colonization & - \\
\hline 9 & 47 & $\mathrm{~F}$ & A & 42 & Ascites & $\begin{array}{l}\text { Surgery to treat } \\
\text { hepatocellular carcinoma }\end{array}$ & Alcoholic liver cirrhosis & Peritonitis & Acinetobacter baumannii \\
\hline 10 & 2 & M & B & 75 & Urine & Acute encephalopathy & Developmental delay & $\begin{array}{l}\text { Asymptomatic } \\
\text { bacteriuria }\end{array}$ & Pseudomonas aeruginosa \\
\hline 11 & 8 & M & B & 92 & $\begin{array}{l}\text { Urine } \\
\text { (catheter) }\end{array}$ & Acute encephalitis & None & $\begin{array}{l}\text { Asymptomatic } \\
\text { bacteriuria }\end{array}$ & - \\
\hline 12 & 2 & $\mathrm{~F}$ & B & 36 & $\begin{array}{l}\text { Tracheal } \\
\text { tube }\end{array}$ & Traffic injury & None & Respiratory infection & $\begin{array}{l}\text { Pseudomonas aeruginosa, } \\
\text { Candida albicans }\end{array}$ \\
\hline 13 & 59 & M & A & 342 & Ascites & Liver transplantation & Fulminant hepatitis B & Intra-abdominal abscess & $\begin{array}{l}\text { Pseudomonas aeruginosa, } \\
\text { Enterococcus faecalis, } \\
\text { Candida parapsilosis }\end{array}$ \\
\hline 14 & 79 & M & A & 39 & Bile & $\begin{array}{l}\text { Surgery to treat } \\
\text { hepatocellular carcinoma } \\
\text { and gastric } \\
\text { cancer }\end{array}$ & $\begin{array}{l}\text { Diabetes mellitus, interstitial } \\
\text { pneumonia, paroxysmal } \\
\text { atrial fibrillation }\end{array}$ & $\begin{array}{l}\text { Intra-abdominal } \\
\text { infection }\end{array}$ & $\begin{array}{l}\text { Enterobacter cloacae, } \\
\text { Enterococcus raffinosus, } \\
\text { Candida glabrata }\end{array}$ \\
\hline 15 & 65 & M & A & 10 & Stool & $\begin{array}{l}\text { Surgery to treat } \\
\text { cholangiocarcinoma }\end{array}$ & $\begin{array}{l}\text { Alcoholic liver cirrhosis, } \\
\text { hypertrophic cardiomyopathy }\end{array}$ & Colonization & - \\
\hline 16 & 63 & $\mathrm{~F}$ & A & 43 & $\begin{array}{l}\text { Urine } \\
\text { (catheter) }\end{array}$ & Liver transplantation & Liver cirrhosis (unknown origin) & Asymptomatic bacteriuria & - \\
\hline
\end{tabular}

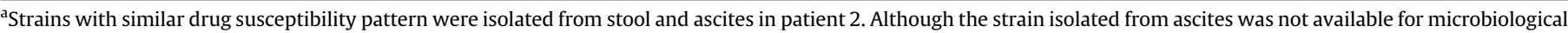
analysis, we deemed that they were the same strain. 
Table 3

Background characteristics of patients and univariate analyses of risk factors for acquisition of IMP-1 metallo- $\beta$-lactamase-producing Klebsiella pneumoniae.

\begin{tabular}{|c|c|c|c|c|c|}
\hline Variables & Cases $(n=16)$ & Controls $(\mathrm{n}=48)$ & OR & $95 \% \mathrm{CI}$ & $\mathrm{P}$ \\
\hline Age (years) & $51.5(6.0-62.0)$ & $59.5(6.5-72.5)$ & 0.9 & $0.8-1.0$ & 0.019 \\
\hline Sex (male/female) & $11 / 5(68.8)$ & $25 / 23(52.1)$ & 2.0 & $0.6-6.4$ & 0.254 \\
\hline ICU/NICU admission within 90 days & $15(93.8)$ & $24(50.0)$ & 13.3 & $1.7-106.1$ & 0.015 \\
\hline Surgery/invasive procedure ${ }^{\mathrm{a}}$ within 90 days & $12(75.0)$ & $32(66.7)$ & 1.7 & $0.4-6.8$ & 0.482 \\
\hline \multicolumn{6}{|l|}{ Comorbidities/conditions } \\
\hline Cerebrovascular disease & $1(6.3)$ & $4(8.3)$ & 0.7 & $0.1-7.4$ & 0.782 \\
\hline Heart disease & $2(12.5)$ & $8(16.7)$ & 0.6 & $0.1-4.4$ & 0.619 \\
\hline Pulmonary disease & $1(6.3)$ & $4(8.3)$ & 0.8 & $0.1-6.7$ & 0.797 \\
\hline Liver disease $^{\mathrm{b}}$ & $12(75.0)$ & $13(27.1)$ & 9.6 & $2.1-45.0$ & 0.004 \\
\hline Renal disease & $1(6.3)$ & $5(10.4)$ & 0.6 & $0.1-5.3$ & 0.621 \\
\hline Diabetes mellitus & $2(12.5)$ & $7(14.6)$ & 0.8 & $0.2-4.3$ & 0.842 \\
\hline Malignancy & $5(31.3)$ & $27(56.3)$ & 0.1 & $0.0-1.1$ & 0.059 \\
\hline Liver transplantation & $8(50.0)$ & $4(8.3)$ & 154.7 & $0.3-75681.9$ & 0.111 \\
\hline Steroids/immunosuppressive agents & $12(75.0)$ & $15(31.3)$ & 6.0 & $1.6-22.6$ & 0.007 \\
\hline Anti-cancer drugs & $1(6.3)$ & $10(20.8)$ & 0.2 & $0.0-2.0$ & 0.172 \\
\hline \multicolumn{6}{|l|}{ Medical devices } \\
\hline Central venous catheter & $11(68.8)$ & $23(47.9)$ & 2.4 & $0.7-8.4$ & 0.158 \\
\hline Tracheal tube & $2(12.5)$ & $3(6.3)$ & 2.3 & $0.3-17.2$ & 0.417 \\
\hline Ventilator & $7(43.8)$ & $10(20.8)$ & 2.7 & $0.8-9.0$ & 0.097 \\
\hline Urinary catheter & $11(68.8)$ & $23(47.9)$ & 2.7 & $0.7-9.7$ & 0.131 \\
\hline Tube feeding & $15(93.8)$ & $26(54.2)$ & 57.0 & $0.4-8554.2$ & 0.114 \\
\hline \multicolumn{6}{|l|}{ Exposure to antibiotics within 90 days } \\
\hline Penicillins & $12(75.0)$ & $19(39.6)$ & 5.9 & $1.2-27.8$ & 0.025 \\
\hline First-generation cephalosporins & $7(43.8)$ & $15(31.3)$ & 1.7 & $0.5-5.3$ & 0.380 \\
\hline Second-generation cephalosporins ${ }^{c}$ & $1(6.3)$ & $6(12.5)$ & 0.4 & $0.0-4.4$ & 0.446 \\
\hline Third-generation cephalosporins & $11(68.8)$ & $11(22.9)$ & 9.4 & $2.0-44.3$ & 0.005 \\
\hline Fourth-generation cephalosporins & $0(0.0)$ & $4(8.3)$ & 0.0 & $0.0-641.2$ & 0.469 \\
\hline Carbapenems & $10(62.5)$ & $14(29.2)$ & 4.3 & $1.3-14.7$ & 0.020 \\
\hline Monobactam & $0(0.0)$ & $0(0.0)$ & - & - & - \\
\hline Fluoroquinolones & $4(25.0)$ & $10(20.8)$ & 1.3 & $0.3-5.0$ & 0.724 \\
\hline Aminoglycosides & $1(6.3)$ & $7(14.6)$ & 0.4 & $0.0-3.3$ & 0.365 \\
\hline Anti-MRSA drugs & $7(43.8)$ & $6(12.5)$ & 12.8 & $1.5-108.9$ & 0.019 \\
\hline Anti-fungal drugs & $8(50.0)$ & $6(12.5)$ & 15.7 & $1.9-130.2$ & 0.011 \\
\hline
\end{tabular}

OR, odds ratio; $\mathrm{CI}$, confidence interval.

Data were expressed as median (interquartile range) or the number (\%).

a Surgery/invasive procedure included neurosurgery, pharyngectomy, esophagectomy, gastrectomy, Hassab's operation, colectomy, gastrostomy, colostomy, intestinal

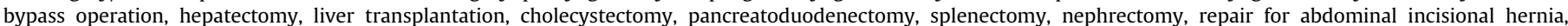
instrument insertion, and endoscopic therapy.

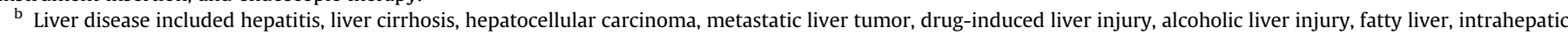
cholestasis, and paucity of interlobular bile duct.

c Cefmetazole was included as a second-generation cephalosporin.

Patient 14, who also developed intra-abdominal infection, was treated with meropenem. These four patients improved and were discharged. Patient 9 with liver failure developed peritonitis caused by both drug-resistant Acinetobacter baumannii and K. pneumoniae producing IMP-1, and subsequently developed complications including pneumonia. Despite receiving antibiotic administration including meropenem, ciprofloxacin, and amikacin the patient ultimately died due to worsening of the general condition.

\subsection{Risk factors for acquiring IMP-1-producing K. pneumoniae}

Details of the case-control study and matched univariate analysis are presented in Table 3. A total of 48 patients were matched as controls to 16 patients with IMP-1-producing K. pneumoniae. In the univariate analysis, age, ICU/neonatal intensive care unit (NICU) within 90 days, liver disease, administration of steroid/immunosuppressive agents, and prior uses of penicillin, third-generation cephalosporins, carbapenems, anti-methicillin-resistant Staphylococcus aureus (MRSA) drugs, and anti-fungal drugs were significant. In the multivariate analysis, seven variables with $P$ values less than 0.05 in the univariate analysis and with regard to comorbidities/ conditions (liver disease, steroids/immunosuppressive agents) and prior antibiotic use (exposure to penicillins, third-generation cephalosporins, carbapenems, anti-MRSA drugs, and anti-fungal drugs) were selected and adjusted. Table 4 shows the matched multivariate analysis of risk factors for acquiring IMP-1-producing strains. Liver disease and previous uses of carbapenems and antifungal drugs were independent factors for acquiring IMP-1producing strains.

\section{Discussion}

We demonstrated that IMP-1 MBL was the unique carbapenemase detected in K. pneumoniae in our hospital, with an incidence of only $1.2 \%$ among all $K$. pneumoniae isolated during the study period. However, several transmissions in the hospital

Table 4

Multivariate analyses of risk factors for acquisition of IMP-1 metallo- $\beta$-lactamaseproducing Klebsiella pneumoniae.

\begin{tabular}{llll}
\hline Variables & OR & $95 \% \mathrm{CI}$ & $\mathrm{P}$ \\
\hline $\begin{array}{l}\text { Comorbidities/conditions } \\
\quad \text { Liver disease }\end{array}$ & 87.3 & $2.2-3401.4$ & 0.017 \\
$\quad \begin{array}{l}\text { Steroids/immunosuppressive agents } \\
\text { Exposure to antibiotics within 90 days }\end{array}$ & - & - & 0.336 \\
$\quad$ Penicillins & - & - & \\
$\quad$ Third-generation cephalosporins & - & - & 0.173 \\
$\quad$ Carbapenems & 14.4 & $1.2-175.4$ & 0.077 \\
$\quad$ Anti-MRSA drugs & - & - & 0.037 \\
Anti-fungal drugs & 27.5 & $1.3-597.1$ & 0.035 \\
\hline
\end{tabular}

OR, odds ratio; $\mathrm{CI}$, confidence interval. 
were suspected because accumulation was observed in three specific wards, and a dendrogram indicated a close relationship between strains isolated in the same ward. The first outbreak of K. pneumoniae producing IMP-1 in Japan was reported from a community hospital in 2007 [10]. Another hospital recently reported a large-scale outbreak of IMP-6-producing Enterobacteriaceae that mainly included Klebsiella species (https://www. niid.go.jp/niid/ja/id/1726-source/drug-resistance/idsc/iasr-news/ 5213-pr4182.html). A number of risk factors for CRE or carbapenemase-producing Enterobacteriaceae (CPE) acquisition have been identified including previous antibiotic use, severity of illness, invasive/indwelling devices, previous or prolonged hospitalization, surgery, liver disease, and organ/stem cell transplantation [23-25]. In this study, liver disease and previous uses of carbapenems and anti-fungal drugs were significant factors for acquiring IMP-1 producers. We could not find the previous report showing that exposure to anti-fungal drugs was a risk factor. It might reflect patient condition which needed anti-fungal therapy due to being under immunosuppression.

Of the five patients who developed infection, one patient showed poor outcome due to severe liver failure and four patients improved. Because they were polymicrobial infection, there is a possibility that IMP-1 producer might have only colonized in those patients.

Specific STs such as ST258 and ST147 are reportedly high-risk clones associated with the international spread of carbapenemaseproducing K. pneumoniae $[9,26]$. Of the seven different STs detected in this study, ST37 and ST443 have been reported to be associated with IMP-6-, KPC-, NDM-, and OXA-48-producing K. pneumoniae [27-32]. However, only a few of those strains were detected in this study. We could not find reports in the literature about ST1471, ST1484, ST1148, or ST2603, which were the most prevalent STs in our hospital. The relationships among them were not close with $<50 \%$ similarity based on PFGE results. Conversely, plasmid incompatibility group was similar among the IMP-1 producers, and the plasmids were transferable. Further studies on those characteristics in major Enterobacteriaceae may be able to reveal plasmid transfer among different bacterial genera or species and its risk factors.

There were some limitations in this study. First, we selected strains for which the MICs for imipenem and/or meropenem were $\geq 1 \mu \mathrm{g} / \mathrm{mL}$. It was difficult to detect all of the CPEs only using drug susceptibility because the MICs of CPE for carbapenems were sometimes $<1 \mu \mathrm{g} / \mathrm{mL}[33,34]$. We could not analyze 32 strains that met the screening criteria but were not available for microbiological analysis. Additionally, since we evaluated only two major carbapenemases, we could not refer to other types of carbapenemases. Regarding the case-control study and analysis of risk factors, because our results might not apply to other institutions, respective hospitals should perform epidemiological evaluations to identify local factors.

In conclusion, we detected 18 carbapanemase-producing K. pneumoniae for 6.5 years in our hospital with IMP-1 MBL being the sole carbapenemase. We should recognize the possibility of transmission in hospital and risk factors for the acquisition of IMP1-MBL-producing K. pneumoniae. Further evaluations are necessary to clarify regional epidemiology.

\section{Authorship statement}

All authors meet the ICMJE authorship criteria.

\section{Conflicts of interest}

The authors have no conflicts of interest to declare

\section{Funding information and acknowledgments}

This study was partially supported by the Health and Labour Sciences Research Grants from Ministry of Health, Labour and Welfare, Japan (H28-Shinkou-Ippan-003) and a grant for Research and development of diagnostic methods and therapies for antimicrobial resistant bacteria from Japan Agency for Medical Research and Development (AMED) (JP18fk0108052). We deeply thank the statisticians, Shuntaro Sato and Shimpei Morimoto, for the assistance with the statistical analyses.

\section{Ethical statement}

This study was approved by the Institutional Review Board of Nagasaki University Hospital (17032719).

\section{Additional information}

This work was presented as a poster at the ASM Microbe 2017 meeting.

\section{References}

[1] Gaynes R, Edwards JR. Overview of nosocomial infections caused by gramnegative bacilli. Clin Infect Dis 2005;41:848-54.

[2] Poirel L, Walsh TR, Cuvillier V, Nordmann P. Multiplex PCR for detection of acquired carbapenemase genes. Diagn Microbiol Infect Dis 2011;70:119-23.

[3] Shindo Y, Ito R, Kobayashi D, Ando M, Ichikawa M, Shiraki A, et al. Risk factors for drug-resistant pathogens in community-acquired and healthcareassociated pneumonia. Am J Respir Crit Care Med 2013:188:985-95.

[4] Tanaka A, Takada T, Kawarada Y, Nimura Y, Yoshida M, Miura F, et al. Antimicrobial therapy for acute cholangitis: Tokyo Guidelines. J Hepatobiliary Pancreat Surg 2007:14:59-67.

[5] Canton R, Akova M, Carmeli Y, Giske CG, Glupczynski Y, Gniadkowski M, et al. Rapid evolution and spread of carbapenemases among Enterobacteriaceae in Europe. Clin Microbiol Infect 2012;18:413-31.

[6] Guh AY, Bulens SN, Mu Y, Jacob JT, Reno J, Scott J, et al. Epidemiology of carbapenem-resistant Enterobacteriaceae in 7 US communities, 2012-2013. J Am Med Assoc 2015;314:1479-87.

[7] Xu Y, Gu B, Huang M, Liu H, Xu T, Xia W, et al. Epidemiology of carbapenem resistant Enterobacteriaceae (CRE) during 2000-2012 in Asia. J Thorac Dis 2015;7:376-85.

[8] Nordmann P, Dortet L, Poirel L. Carbapenem resistance in Enterobacteriaceae: here is the storm! Trends Mol Med 2012;18:263-72.

[9] Pitout JD, Nordmann P, Poirel L. Carbapenemase-producing Klebsiella pneumoniae, a key pathogen set for global nosocomial dominance. Antimicrob Agents Chemother 2015:59:5873-84.

[10] Fukigai S, Alba J, Kimura S, Iida T, Nishikura N, Ishii Y, et al. Nosocomial outbreak of genetically related IMP-1 beta-lactamase-producing Klebsiella pneumoniae in a general hospital in Japan. Int J Antimicrob Agents 2007;29:306-10.

[11] Livermore DM, Woodford N. Carbapenemases: a problem in waiting? Curr Opin Microbiol 2000;3:489-95.

[12] Tojo M, Tada T, Shimojima M, Tanaka M, Narahara K, Miyoshi-Akiyama T, et al. Dissemination in Japan of multidrug-resistant Pseudomonas aeruginosa isolates producing IMP-type metallo-beta-lactamases and AAC(6')-Iae/AAC(6')Ib. J Infect Chemother 2014;20:586-8.

[13] Japan nosocomial infections surveillance (JANIS). https://janis.mhlw.go.jp/.

[14] Shigemoto N, Kuwahara R, Kayama S, Shimizu W, Onodera M, Yokozaki M, et al. Emergence in Japan of an imipenem-susceptible, meropenem-resistant Klebsiella pneumoniae carrying blaIMP-6. Diagn Microbiol Infect Dis 2012;72: 109-12.

[15] Clinical and Laboratory Standards Institute (CLSI). Performance standards for antimicrobial susceptibility testing. CLSI supplement M100. Wayne, PA: Clinical and Laboratory Standards Institute; 2018.

[16] Yano H, Kuga A, Okamoto R, Kitasato H, Kobayashi T, Inoue M. Plasmidencoded metallo- $\beta$-lactamase (IMP-6) conferring resistance to carbapenems, especially meropenem. Antimicrob Agents Chemother 2001;45:1343-8.

[17] Diancourt L, Passet V, Verhoef J, Grimont PA, Brisse S. Multilocus sequence typing of Klebsiella pneumoniae nosocomial isolates. J Clin Microbiol 2005;43: 4178-82.

[18] Japanese Association of Medical Technologists. Rinshokensagishinotameno idenshi senshokutaikensa gaidobukku. 2003 (in Japanese).

[19] Tenover FC, Arbeit RD, Goering RV, Mickelsen PA, Murray BE, Persing DH, et al. Interpreting chromosomal DNA restriction patterns produced by pulsed-field gel electrophoresis: criteria for bacterial strain typing. J Clin Microbiol 1995;33: 2233-9.

[20] Harris AD, Samore MH, Lipsitch M, Kaye KS, Perencevich E, Carmeli Y. Controlgroup selection importance in studies of antimicrobial resistance: examples 
applied to Pseudomonas aeruginosa, Enterococci, and Escherichia coli. Clin Infect Dis 2002;34:1558-63.

[21] Hayakawa K, Miyoshi-Akiyama T, Kirikae T, Nagamatsu M, Shimada K, Mezaki K, et al. Molecular and epidemiological characterization of IMP-type metallo- $\beta$-lactamase-producing Enterobacter cloacae in a Large tertiary care hospital in Japan. Antimicrob Agents Chemother 2014;58:3441-50.

[22] Lubbert C, Becker-Rux D, Rodloff AC, Laudi S, Busch T, Bartels M, et al. Colonization of liver transplant recipients with KPC-producing Klebsiella pneumoniae is associated with high infection rates and excess mortality: a casecontrol analysis. Infection 2014;42:309-16.

[23] Salomao MC, Guimaraes T, Duailibi DF, Perondi MBM, Letaif LSH, Montal AC, et al. Carbapenem-resistant Enterobacteriaceae in patients admitted to the emergency department: prevalence, risk factors, and acquisition rate. J Hosp Infect 2017;97:241-6.

[24] ECDC. Risk assessment on the spread of carbapenemase-producing Enterobacteriaceae (CPE) through patient transfer between healthcare facilities, with special emphasis on cross-border transfer. 2011. Stockholm.

[25] French CE, Coope C, Conway L, Higgins JP, McCulloch J, Okoli G, et al. Control of carbapenemase-producing Enterobacteriaceae outbreaks in acute settings: an evidence review. J Hosp Infect 2017;95:3-45.

[26] Chen L, Mathema B, Chavda KD, DeLeo FR, Bonomo RA, Kreiswirth BN. Carbapenemase-producing Klebsiella pneumoniae: molecular and genetic decoding. Trends Microbiol 2014;22:686-96.

[27] Liu Y, Wan LG, Deng Q, Cao XW, Yu Y, Xu QF. First description of NDM-1-, KPC2-, VIM-2- and IMP-4-producing Klebsiella pneumoniae strains in a single Chinese teaching hospital. Epidemiol Infect 2015;143:376-84.
[28] Ohno Y, Nakamura A, Hashimoto E, Matsutani H, Abe N, Fukuda S, et al. Molecular epidemiology of carbapenemase-producing Enterobacteriaceae in a primary care hospital in Japan, 2010-2013. J Infect Chemother 2017;23: 224-9.

[29] Seki LM, Pereira PS, de Souza Mda P, Conceicao Mde S, Marques EA, Porto CO et al. Molecular epidemiology of KPC-2- producing Klebsiella pneumoniae isolates in Brazil: the predominance of sequence type 437. Diagn Microbiol Infect Dis 2011;70:274-7.

[30] Uz Zaman T, Aldrees M, Al Johani SM, Alrodayyan M, Aldughashem FA Balkhy HH. Multi-drug carbapenem-resistant Klebsiella pneumoniae infection carrying the OXA-48 gene and showing variations in outer membrane protein 36 causing an outbreak in a tertiary care hospital in Riyadh, Saudi Arabia. Int J Infect Dis 2014;28:186-92.

[31] Yang J, Ye L, Guo L, Zhao Q, Chen R, Luo Y, et al. A nosocomial outbreak of KPC2-producing Klebsiella pneumoniae in a Chinese hospital: dissemination of ST11 and emergence of ST37, ST392 and ST395. Clin Microbiol Infect 2013;19: E509-15.

[32] Zhu J, Sun L, Ding B, Yang Y, Xu X, Liu W, et al. Outbreak of NDM-1-producing Klebsiella pneumoniae ST76 and ST37 isolates in neonates. Eur J Clin Microbio Infect Dis 2016:35:611-8.

[33] Giske CG, Martinez-Martinez L, Canton R, Stefani S, Skov R, Glupczynski Y et al. EUCAST guideline for the detection of resistance mechanisms and specific resistances of clinical and/or epidemiological importance. 2013.

[34] Segawa T, Matsui M, Suzuki M, Tsutsui A, Kuroda M, Shibayama K, et al Utilizing the Carba NP test as an indicator of expression level of carbapenemase genes in Enterobacteriaceae. J Microbiol Methods 2017;133:35-9. 